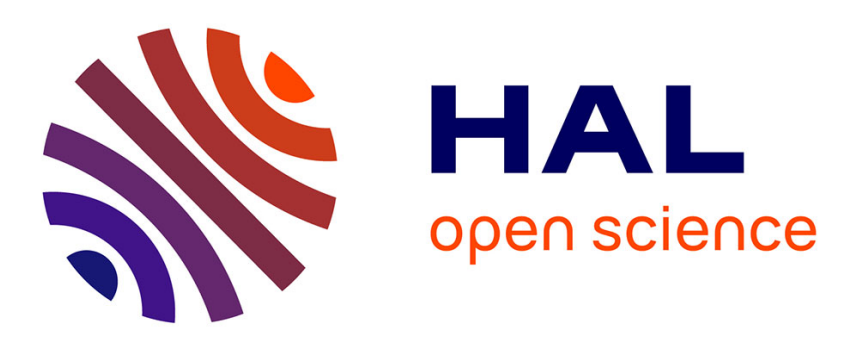

\title{
Oxydation comparée du niobium et d'un alliage (P 353 M) à base de niobium \\ E. Fries, C. Marhic
}

\section{To cite this version:}

E. Fries, C. Marhic. Oxydation comparée du niobium et d'un alliage (P $353 \mathrm{M})$ à base de niobium. Revue de Physique Appliquée, 1970, 5 (3), pp.519-522. 10.1051/rphysap:0197000503051900 . jpa00243423

\section{HAL Id: jpa-00243423 https://hal.science/jpa-00243423}

Submitted on 1 Jan 1970

HAL is a multi-disciplinary open access archive for the deposit and dissemination of scientific research documents, whether they are published or not. The documents may come from teaching and research institutions in France or abroad, or from public or private research centers.
L'archive ouverte pluridisciplinaire HAL, est destinée au dépôt et à la diffusion de documents scientifiques de niveau recherche, publiés ou non, émanant des établissements d'enseignement et de recherche français ou étrangers, des laboratoires publics ou privés. 


\title{
OXYDATION COMPARÉE DU NIOBIUM ET D'UN ALLIAGE (P 353 M) A BASE DE NIOBIUM
}

\author{
E. FRIES, C. MARHIC
}

Laboratoire de Rayons X du C. N. R. S., 92, Bellevue

Résumé. - Etude par diffraction de rayons $X$ de l'oxydation comparée du Niobium pur et d'un alliage à base de Niobium de composition suivante :

$$
\begin{array}{cccccc} 
& \mathrm{W} & \mathrm{V} & \mathrm{Ti} & \mathrm{Zr} & \mathrm{C} \\
\mathrm{Nb} \text { élément de base } & 10 \% & 1,5 \% & 0,5 \% & 3 \% & 0,25 \% \\
& 12 \% & 2 \% & 0,7 \% & 4 \% & 0,40 \%
\end{array}
$$

L'oxydation a été effectuée à l'air pour des températures variant de $800^{\circ} \mathrm{C}$ à $950^{\circ} \mathrm{C}$ et des temps s'échelonnant entre $1 / 2 \mathrm{~h}$ et $100 \mathrm{~h}$.

Dans les deux cas (niobium et alliage) on a mis en évidence la présence de trois formes de l'oxyde $\mathrm{Nb}_{2} \mathrm{O}_{5}$ :

- une forme de basse température $\mathrm{B}$. T.

- une forme de moyenne température M. T. ;

- une forme de haute température $H$. $T$.

Le Niobium et l'alliage P $353 \mathrm{M}$ ont un comportement globalement identique quant à la nature des phases et à leur température d'apparition. L'oxydation de l'alliage est cependant retardée par rapport à celle du Niobium pur. On observe aussi dans le cas de l'alliage :

- une stabilisation de la forme B. T. ;

- une apparition plus tardive des formes M. T. et H. T.

et une croissance plus lente de ces phases M. T. et H. T.

Abstract. - X ray diffraction study of the oxidation of pure Niobium compared whit an alloy of the following composition :

$$
\begin{array}{cccccc} 
& \mathrm{W} & \mathrm{V} & \mathrm{Ti} & \mathrm{Zr} & \mathrm{C} \\
& 10 \% & 1.5 \% & 0.5 \% & 3 \% & 0.25 \% \\
\text { Nb main component } & \text { to } & \text { to } & \text { to } & \text { to } & \text { to } \\
& 12 \% & 2 \% & 0.7 \% & 4 \% & 0.40 \%
\end{array}
$$

Oxidation has been achieved in atmospheric conditions at temperatures ranging from $800{ }^{\circ} \mathrm{C}$ to $950^{\circ} \mathrm{C}$ and for periods of time ranging from $1 / 2 \mathrm{~h}$ to $100 \mathrm{~h}$.

In both cases (Niobium and alloy) 3 forms of the Niobium oxide $\mathrm{Nb}_{2} \mathrm{O}_{5}$ have appeared :

- a low temperature form B. T. ;

- a medium temperature form $\mathrm{M}$. $\mathrm{T}$.

- and a high temperature form $\mathbf{H}$. T.

The Niobium and the P $353 \mathrm{M}$ alloy behave most identically as to the different phases and their temperatures of appearance. Oxidation of the alloy is however delayed if compared with pure Niobium. With the alloy we can observed :

- a stabilization of the B. T. form ;

- a delayed appearance of the M.'T. and H. T. forms and a slower growth of these M. T. and $H$. T. forms.

Cette étude a été conduite dans le but de comparer le comportement du niobium pur et d'un alliage à base de niobium (P $353 \mathrm{M})$ vis-à-vis de l'oxygène de l'air pour une gamme de températures variant de $800^{\circ} \mathrm{C}$ à $950^{\circ} \mathrm{C}$ et pour des durées s'échelonnant entre $1 / 2 \mathrm{~h}$ et $100 \mathrm{~h}$.

La composition de cet alliage est la suivante :

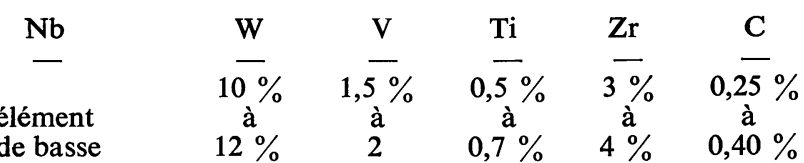

Notre objectif était de comparer dans les deux cas l'apparition, le développement et les transformations des diverses formes de l'oxyde $\mathrm{Nb}_{2} \mathrm{O}_{5}$ en fonction du temps et de la température afin de mettre en évidence l'influence des éléments d'addition introduits dans l'alliage.

L'identification des différentes formes de l'oxyde
$\mathrm{Nb}_{2} \mathrm{O}_{5}$ a été faite par diffraction de rayons $\mathrm{X}$ au moyen d'un diffractomètre comportant un échantillon vertical. Une chambre chauffante dans l'air permet facilement d'observer le commencement de l'oxydation ; mais lorsque celle-ci se poursuit, l'échantillon s'effrite, se boursoufle et ne présente plus la surface plane nécessaire à une manipulation correcte. Une identification précise des oxydes in situ est d'autre part difficile par suite d'orientations préférentielles dans la couche d'oxyde. Au-delà d'une heure de chauffage, nous avons donc réalisé l'oxydation dans un four Chévenard à la pression atmosphérique, les échantillons du métal et de l'alliage étant toujours placés côte à côte dans le four ; les poudres d'oxydes recueillies ont été examinées ensuite en diffractométrie de rayons $\mathrm{X}$.

Caractérisation des divers oxydes. - Parmi les nombreuses formes connues [1] [2] du pentoxyde de niobium $\mathrm{Nb}_{2} \mathrm{O}_{5}$, nous avons mis en évidence aussi 


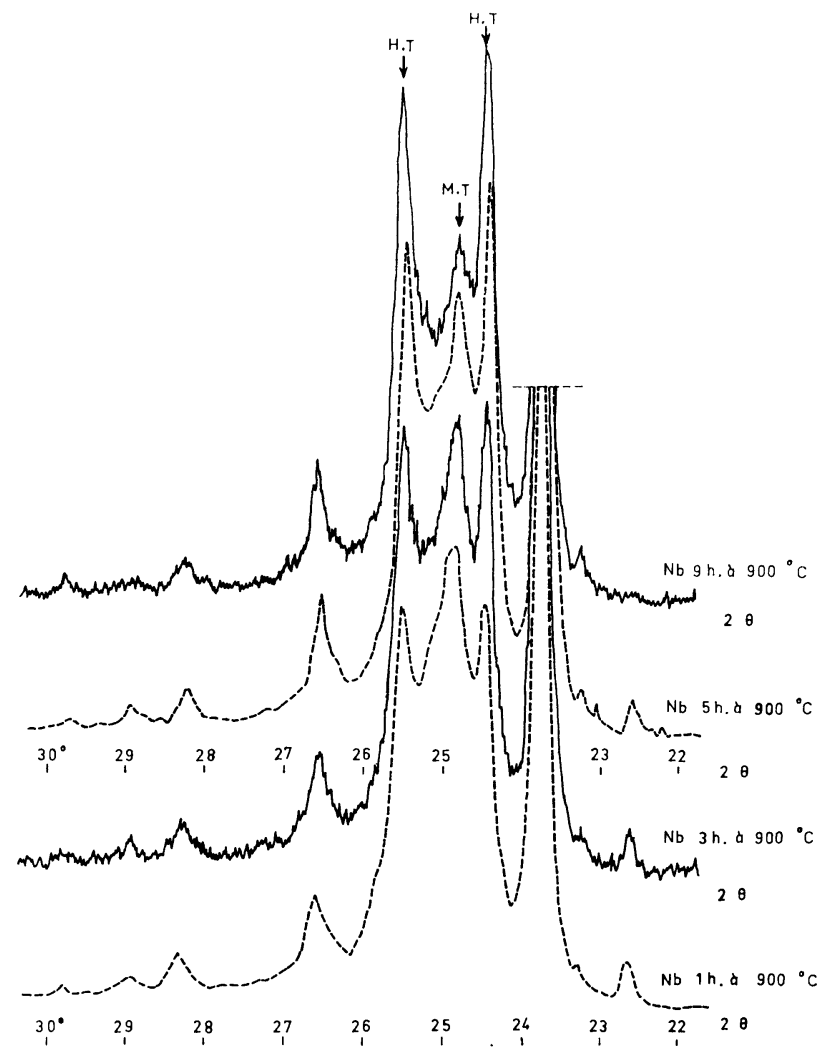

Fig. 1. - Niobium, $1 \mathrm{~h}, 3 \mathrm{~h}, 5 \mathrm{~h}, 9 \mathrm{~h}$, à $900^{\circ} \mathrm{C}$. Transformation continue, M. T. $\rightarrow$ H. T.

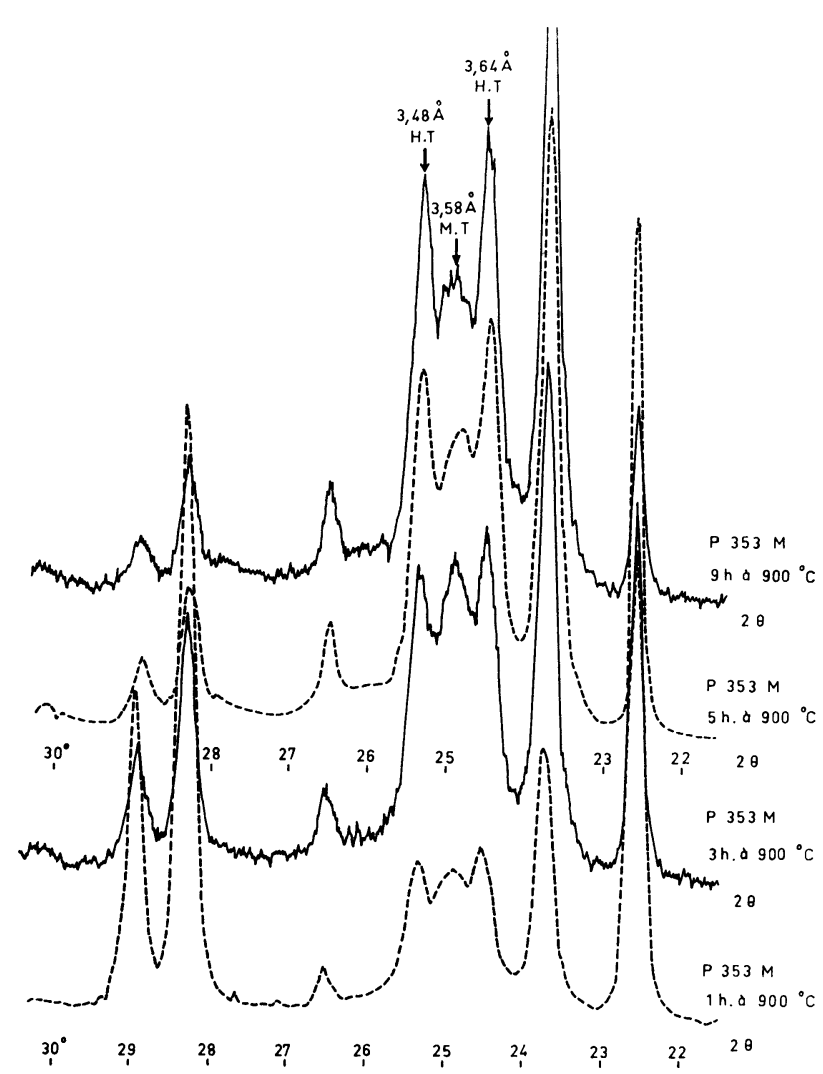

Fig. 2. - P $353 \mathrm{M}, 1$ h, $3 \mathrm{~h}, 5 \mathrm{~h}, 9 \mathrm{~h}$, à $900^{\circ} \mathrm{C}$. Transformation M. T. $\rightarrow$ H. T.

TABLEAU RÉCAPITULATIF

Oxydation à l'air du niobium et de l'alliage P $353 \mathrm{M}$

\section{Niobium}

tempér./temps

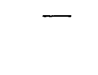

$800^{\circ} \mathrm{C}$

$850^{\circ} \mathrm{C}$

(2000

$900{ }^{\circ} \mathrm{C}$

P 353 M

$800^{\circ} \mathrm{C}$

$850^{\circ} \mathrm{C}$

$900{ }^{\circ} \mathrm{C}$
$1 / 2 \mathrm{~h}$

B. T.

B. T.

B. T. $\# 0$

M. T. $\nearrow$

H. T. (f)

B. T.

B. T.

$\frac{\text { B. T. }}{\text { M. T. }}$

B. T.

$\frac{\text { B. T. }}{\text { M. T. (f) }}$

H. T. (f)

B. T. $\# 0$

M. T. $\nearrow(\mathrm{r})$

H. T. $\nearrow(\mathrm{r})$

B. T.

$1 \mathrm{~h}$

$5 \mathrm{~h}$

B. T.

M. T. (f)

H. T. (f)

B. T. \

M. T. $\nearrow$

H. T.

B. T. \# 0

M. T. \# cte

H. T.

B. T.

B. T.

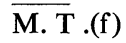

H. T. (f)

B. $T$.

M. T. $\nearrow$

B. T. \

H. T. $\nearrow$
M. T. \# cte

H.T. $\nearrow$
$9 \mathrm{~h}$

B. T.

M. T. $\nearrow$

H. T. $\nearrow$

B. T. $\# 0$

M. T. \# cte

H. T. $\nearrow$

M. T. \

H. T. $\nearrow$

B. $T$.

M. T. $\nearrow$

H. T. $\nearrow$

B. T. \

M. T. \# cte

H. T.
$23 \mathrm{~h}$

B. T. \

M. T. $\nearrow$

H. T. $\nearrow$

M. T. $\searrow(\mathrm{L})$

H. T. $\nearrow$

M. T. \

H. T. $\nearrow$

B. T.

M. T. (f)

B. T.

$90 \mathrm{~h}$

$120 \mathrm{~h}$

M. T. \# cte

H. T. \# cte

M. T. \# cte

H. T. \# cte

M. T. \(L) M. T. $\searrow$

H. T. $\nearrow$ H.T. $\nearrow$

M. T. $\searrow \quad$ M. T. $\searrow$

H. T. $\nearrow$ H. T. $\nearrow$

M. T. $\nearrow(\mathrm{L})$ M. T.

H. T. (f) H. T. $\nearrow$ (L) H. T. $\nearrow$

B. T. ป

M. T. $\nearrow$

H. T. $\nearrow$

M. T. \# cte

M. T. \(L)

B. T. $\# 0$

M. T. \# cte

H. T. $\nearrow$
H. T. $\nearrow$

H. T. $\nearrow(\mathrm{L})$

M. T. \(L) M. T. $\searrow(L)$

La phase prédominante dans chaque cas a été soulignée en trait plein.

$$
\begin{aligned}
& \text { \# } 0 \text { : traces. } \\
& \text { \# cte : approximativement constant. } \\
& \text { (f) : quantité faible. }
\end{aligned}
$$

$\nearrow(\mathrm{L})$ ou $\searrow(\mathrm{L})$ : croissance ou décroissance lente.

$\nearrow(\mathrm{r})$ ou $\searrow(\mathrm{r})$ : croissance ou décroissance rapide. 
bien dans le cas de l'alliage que dans celui du niobium pur :

- une forme que nous désignerons comme forme de basse température (B. T.),

- une forme que nous désignerons comme forme de moyenne température (M. T.),

- une forme que nous désignerons comme forme de haute température (H. T.).

Certains auteurs ont désigné par $\alpha$ la forme de basse température et $\beta$ la forme de haute température, d'autres ont adopté une dénomination inverse ou même très différente [2] ; dans le cas présent, il était inutile de recourir à ces nomenclatures.

CAS DU NIOBIUM PUR. - Les trois formes d'oxyde observées correspondent aux fiches A. S. T. M. suivantes :

$\mathrm{Nb}_{2} \mathrm{O}_{5}$ B. T. \{ Système inconnu $\mathrm{n}^{0}$ 5-0352

$\mathrm{Nb}_{2} \mathrm{O}_{5} \mathrm{~B}$. T. Système pseudohexagonal $\mathrm{n}^{0}$ 7-61

$\mathrm{Nb}_{2} \mathrm{O}_{5}$ M. T. Système monoclinique $\mathrm{n}^{0} 15-166$ $\mathrm{Nb}_{2} \mathrm{O}_{5}$ H. T. Système monoclinique $\mathrm{n}^{0} 16-53$

La forme M. T. est considérée parfois comme une modification moins ordonnée de la forme $H$. T. Elle présente de nombreuses raies de diffraction semblables à celles de la forme $\mathbf{H}$. T. mais s'en différencie par 3 raies supplémentaires à :

$$
\begin{aligned}
4,88 \AA & \text { (intensité faible) } \\
3,57 \AA & \text { (intensité forte) } \\
\text { et } 2,05 \AA & \text { (intensité moyenne). }
\end{aligned}
$$

CAS de l'alliage P 353 M. - Si à basse température on trouve pour le niobium et pour l'alliage P 353 M la même variété d'oxyde B. T., on note une déformation du réseau des phases $M$. T. et $H$. T. dans le cas de l'alliage où les raies de diffraction $\mathrm{X}$ sont élargies et légèrement déplacées : certaines raies présentent une faible variation de distance réticulaire (de l'ordre de $0,02 \AA$ ) qui est reproductible. Il n'a pas été possible cependant de mettre en évidence d'oxyde mixte ou de phases différentes dans le cas de l'alliage.

Comme dans le cas du niobium pur, on constate pour l'alliage un changement continu de la forme M. T. en la forme H. T. en fonction du temps quelle que soit la température de chauffage :

$$
800^{\circ}-850^{\circ}-900^{\circ}-950^{\circ} \mathrm{C} \text {. }
$$

Les figures 1 et 2 mettent en évidence cette évolution pour des chauffages successifs de :

$$
1 \mathrm{~h}, 3 \mathrm{~h}, 5 \mathrm{~h}, 9 \mathrm{~h} \text { à } 900^{\circ} \mathrm{C} \text {. }
$$

La raie à $3,58 \AA$ (240 $80=2 \theta$ avec anticathode de cuivre) est caractéristique de la forme M. T. tandis que les raies à $3,48 \AA\left(25^{\circ} 54=2 \theta\right)$ et $3,64 \AA$ $\left(24^{\circ} 40=2 \theta\right)$ qui l'entourent sont caractéristiques de la forme $H$. $T$.

Comparaison du comportement du niobium et de l'alliage P $353 \mathrm{M}$. - Le tableau précédent résume nos résultats à $800^{\circ} \mathrm{C}-850^{\circ} \mathrm{C}-900^{\circ} \mathrm{C}$.

Il n'a pas été nécessaire d'effectuer d'étude systéma- tique à $950^{\circ} \mathrm{C}$ quelques expériences ayant seulement mis en évidence une progression plus rapide des

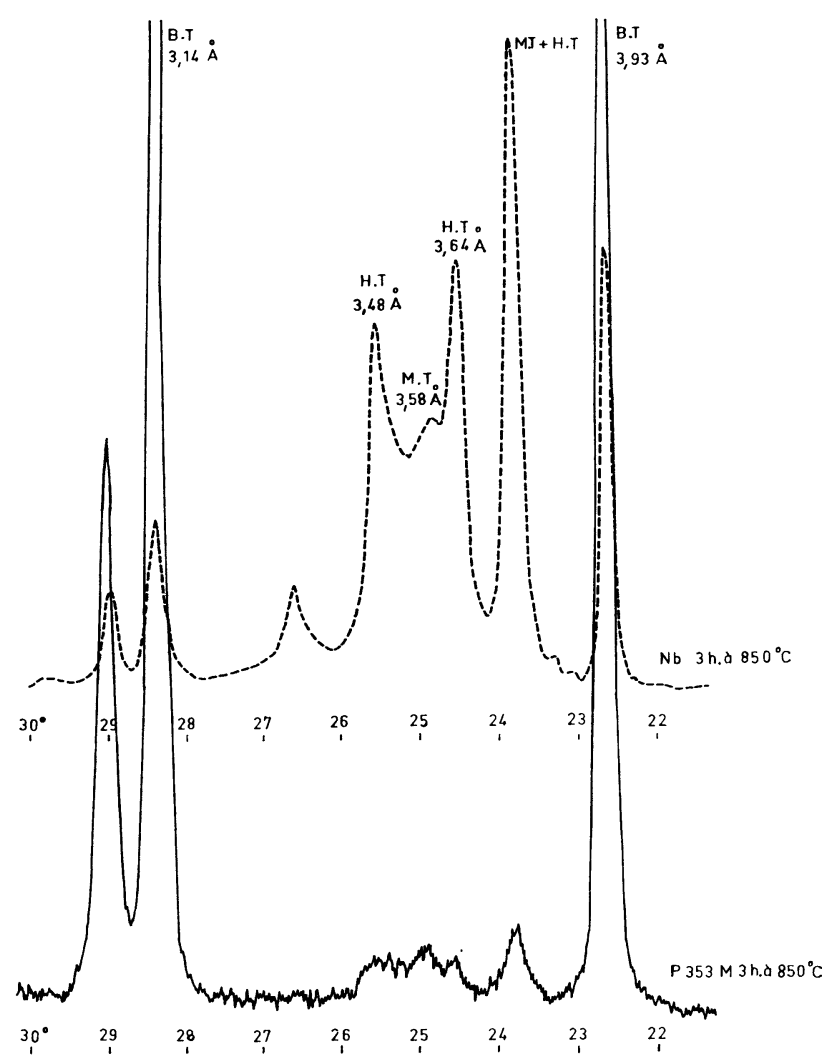

Fig. 3. - Nb et $\mathrm{P} 353 \mathrm{M}, 3 \mathrm{~h}$ à $850^{\circ} \mathrm{C}$. Evolution relative des phases B. T., M. T., H. T.

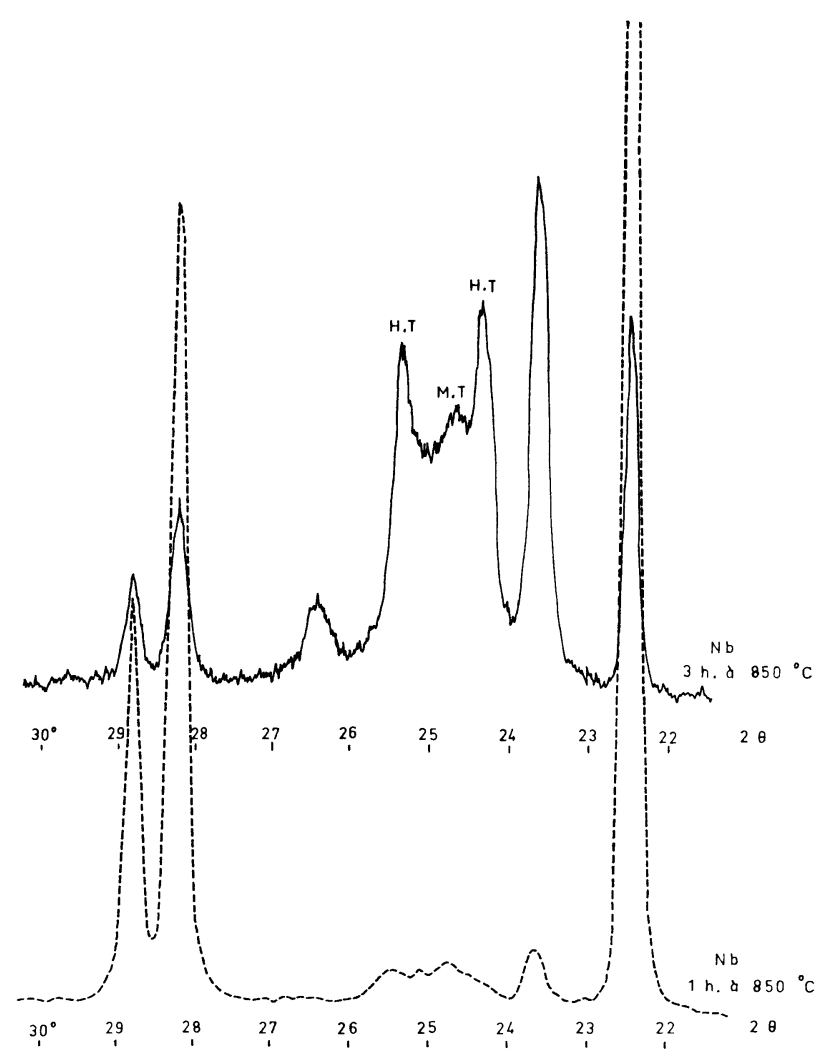

Fig. 4. - Nb, $1 \mathrm{~h}$ et $3 \mathrm{~h}$, à $850^{\circ} \mathrm{C}$. Croissance des phases, M. T. et H. T. 
transformations polymorphiques. D'autre part, nous n'avons pas constaté de variation particulière des distances réticulaires des trois formes d'oxyde selon la température ou la cinétique de l'oxydation pour le niobium et l'alliage.

- D'une manière générale le niobium et l'alliage P $353 \mathrm{M}$ ont un comportement globalement identique quant à la nature des phases et à leur température d'apparition.

Au cours d'une montée en température de $400^{\circ} \mathrm{C} / \mathrm{h}$, on observe :

a) vers $500{ }^{\circ} \mathrm{C}$ une apparition rapide de $\mathrm{Nb}_{2} \mathrm{O}_{5}$ B. T.

b) vers $900^{\circ} \mathrm{C}$ :

1) Une décroissance de la phase $B$. T. et apparition des phases $\mathrm{Nb}_{2} \mathrm{O}_{5} M$. T. et $\mathrm{H}$. T.

2) Puis au cours du temps une croissance de la phase $H$. T. avec diminution de la phase $M$. T. et disparition de la phase $B$. T.

Les vitesses de ces diverses transformations dépendent de la température à $\pm 5^{\circ} \mathrm{C}$, d'où la nécessité d'un bon contrôle de celle-ci.

D'autre part, une bonne homogénéisation de la poudre est nécessaire pour la reproductibilité des phénomènes. Nous avons en effet constaté un retard du développement des phases $M$. T. et $H$. T. dans la partie de la couche d'oxyde la plus proche du métal par rapport à la partie plus externe.

- Dans le cas de l'alliage, les phénomènes sont cependant retardés et leur évolution est plus lente. -

On observe :

1) Une stabilisation de la forme B. T. par rapport au niobium pur.

La figure 3 met en évidence la disparition beaucoup plus rapide de la phase $B$. T. dans le cas du métal pur, les raies caractéristiques de cette phase étant à 3,93 $\AA$ et $3,14 \AA$.

2) Une apparition plus tardive des formes $M . T$. et $\mathbf{H}$. T. dans le cas de l'alliage.

Ce phénomène apparaît nettement sur la figure précédente $\mathrm{n}^{\circ} 3$, les raies caractéristiques de ces formes étant à $3,58 \AA$ (forme $M$. T.) et $3,64 \AA$ et $3,48 \AA$ (forme H. T.).

3) Une croissance plus lente des phases $M$. T. et H. T. dans le cas de l'alliage.

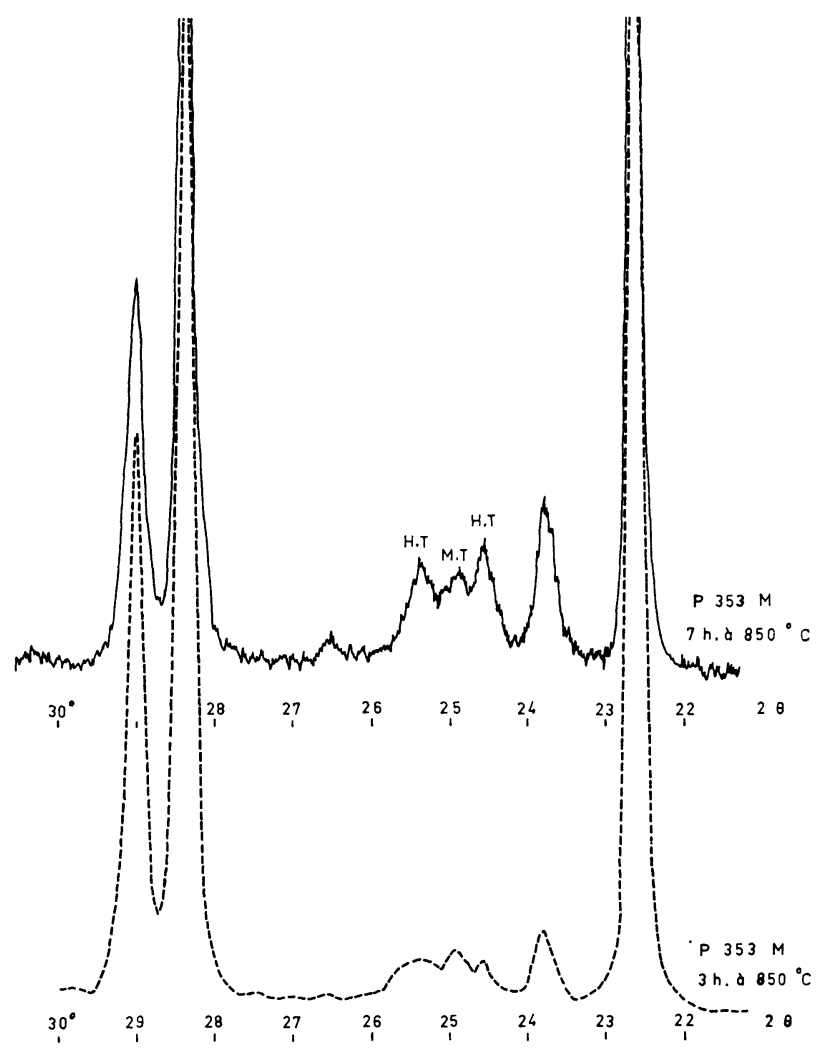

Fig. 5. - P $353 \mathrm{M}, 3 \mathrm{~h}$ et $7 \mathrm{~h}$, à $850^{\circ} \mathrm{C}$. Croissance des phases, M. T. et H. T.

La figure 4 montre la croissance des phases M. T. et $\mathrm{H}$. T. pour le niobium entre $1 \mathrm{~h}$ et $3 \mathrm{~h}$ à $850^{\circ} \mathrm{C}$, la figure 5 celle de ces mêmes phases pour l'alliage $\mathrm{P} 353 \mathrm{M}$ entre $3 \mathrm{~h}$ et $7 \mathrm{~h}$ à $850^{\circ} \mathrm{C}$.

En conclusion. - Il semble que l'oxydation nettement retardée de l'alliage P 353 M par suite de l'effet bénéfique des éléments d'addition au niobium, résulte de la stabilisation de la forme B. T. ou d'une évolution plus lente de la transformation :

$$
\mathrm{Nb}_{2} \mathrm{O}_{5} \text { B. T. } \rightarrow \begin{cases}\mathrm{Nb}_{2} \mathrm{O}_{5} & \text { M. T. } \\ \mathrm{Nb}_{2} \mathrm{O}_{5} & \text { H. T. }\end{cases}
$$

Cette différence de comportement pourrait peut être résulter d'une diffusion plus lente de l'oxygène dans le réseau de l'alliage que dans celui du niobium, comme le suggèrent certains auteurs [3].

\section{Bibliographie}

[1] ZvinchuK, Soviet Physics Cryst., 1958, 3, 750.

[2] SChäfer, Gruehn et Schulte, Angew. Chem., internat. Edit., 1966, 5, 40 à 52. Revue critique et classifi- cation des diverses formes de $\mathrm{Nb}_{2} \mathrm{O}_{5}$ (81 références).

[3] Arkharov, Girasimov et Ushkova, Fizika Metallov i Metallovedenie, 1961, 12, (5) 761-763. 